\title{
LOW-RESOLUTION SPHERICAL HARMONICS MODELS IN APPLICATION TO QUASI-QUADRIC PARTICLE SHAPES
}

\author{
Urtė RADVILAITĖ a Álvaro RAMÍREZ-GÓMEZ ${ }^{\mathrm{b}}$, Arūnas JARAS ${ }^{\mathrm{a}}$, \\ Rimantas KAČIANAUSKAS ${ }^{a}$, Dainius RUSAKEVIČIUS ${ }^{a}$ \\ ${ }^{a}$ Department of Strength of Materials and Engineering Mechanics, \\ Vilnius Gediminas Technical University, Sauletekio al. 11, LT-10223 Vilnius, Lithuania \\ ${ }^{b}$ Department of Mechanical, Chemical and Industrial Design Engineering, \\ Technical University of Madrid, Ronda de Valencia, 3, 28012 Madrid, Spain
}

Received 04 November 2016; accepted 30 November 2016

\begin{abstract}
In this paper a numerical analysis was performed developing low-resolution spherical harmonics (LRSH) models in order to describe particle shapes. The potential of LRSH, limited by the expansion degree $\mathrm{L} \leq 3$, to describe quasi-regular particle shapes was explored. The term "quasi" is used hereafter to indicate the monomeric, almost regular shaped, particle described by a single continuous function. This approach reflects the shape of a major part of soil minerals. It was shown, that even the simplest case of the suggested low-resolution harmonics technique with $\mathrm{L}=1$ showed sufficient accuracy. The main drawback of the suggested approach was that the low-resolution harmonics yield particle shapes with nearly sharp angles, therefore, enhanced analysis of local surface curvatures becomes necessary. An application using quasi-ellipsoidal particles is enclosed.
\end{abstract}

Keywords: soil, particle, quasi-quadric shape, spherical harmonics, curvature, contact.

\section{Introduction}

Characterization of geomaterials and soils plays an important role in many areas of civil and geotechnical engineering. It is obvious that these materials should be treated as discrete elements, and that the mechanical properties of these materials depend on properties of individual particles, thereby, some of them such as packing density, flowability, dilatancy, crushability, friction, etc., are highly contributed by the particle shape. The influence of the particle shape in the behaviour of aggregated materials is considered by Santamarina and Cho (2004), Kock and Huhn (2007) and Cleary (2008). Basic concepts and different measures used to classify particle shapes are given in review papers (Nouguier-Lehon et al. 2003; Blott, Pye 2008; Bullard, Garboczi 2013; Parafiniuk et al. 2014).
It is obvious that methods that accurately describe non-spherical shapes are still needed for both technological and computational purposes. On the other hand, accuracy is not the unique requirement, different approaches based on the compromise between accuracy and simplicity have been proposed for the description of particle shapes, especially for its application with the Discrete Element Method (DEM). A critical review of recent developments in DEM with advances in the formulation and the implementation of particle shape models is presented by Lu et al. (2015), where properties of the simplest shapes (ellipsoids, super-quadrics, polyhedrons and composite multi-sphere models) are reviewed. Concerning complex shapes, broad variety of universal methods ranging from purely numeric discrete function representa-

Corresponding author:

Á. Ramírez-Gómez E-mail: alvaro.ramirez@upm.es 
tion (DFR) (Johnson, Williams 2009) up to analytical continuous function representation (CFR) methods are also explored (Lu et al. 2015).

In many situations, an analytical description of particles is more advantageous compared to composite or purely numeric approaches. Garboczi (2002) was probably the first employing the spherical harmonics ( $\mathrm{SH})$ technique to mathematically describe in threedimensions particle shapes applying it to aggregates used in concrete. A review of computational methods including the radius method, gradient method, Fourier series and spherical harmonic series was presented by Masad et al. (2005). In this study, a number of computational methods were utilized to describe the surface characteristics (form, angularity and texture) of particles. Subsequently, these methods were used to analyse images captured by non-destructive X-ray computer tomography. The SH method was successfully applied in the description of sand (Zhou et al. 2015) or even of quite specific lunar regolith particles (Garboczi 2011). Application of $\mathrm{SH}$ to describe agricultural grains is given in Radvilaitè et al. (2016). Illustrations of very complex shapes can be found in Mousa et al. (2008) while various shape indicators described in terms of SH were considered in Feinauer et al. (2015).

The major part of the above mentioned applications addressed the suitability of SH technique to precisely describe particle shapes. Desired accuracy of semi-analytical SH approximation is controlled by a suitable value of expansion degree $L$. It was found, however, see Feinauer et al. (2015), Zhou et al. (2015), that essential characteristics of the shape geometry are captured by a limited number of expansion terms, while the residual terms are responsible for the detailed description of the particle surface. Basic features of spherical harmonic modelling methodology, addressing the applicability of low-resolution spherical harmonics to describe symmetric particles with sharp edges shape were considered by Radvilaite et al. (2017).

This paper addresses the development of numerical tools based on low-resolution spherical harmonics (LRSH) models limited by the expansion degree $L \leq 3$ that are applied to description of quasi-quadric shapes. The term "quasi" is used hereafter to indicate the monomeric almost regular shaped particle described by the single continuous function. The suitability of the LRSH to describe the shape of quasi-ellipsoids including an evaluation of normal contacts is demonstrated.

\section{Theoretical background}

Spherical harmonics (SH) represent a complete set of angular functions in spherical coordinates, where the position of a point is defined by the polar radius $r$ and two polar and azimuthal spherical angles, $\theta$ and $\varphi$, respectively. Thereby, azimuthal angle $\theta$ is counted from Cartesian axis $\mathrm{Oz}$. This approach provides a convenient way to characterize the surface of a complex object. An arbitrary surface function $R(\theta, \varphi)$ defined in angular spherical coordinates $\theta$ and $\varphi$ can be expanded using spherical harmonics series. In applications, it is approximated by function $F(\theta, \varphi)$ which is limited to a finite number of harmonics as follows:

$$
F(\theta, \varphi)=\sum_{l=0}^{L} \sum_{m=-l}^{l} a_{l}^{m} Y_{l}^{m}(\theta, \varphi),
$$

where $Y_{l}^{m}$ denotes spherical harmonics of degree $l$ and order $m(0 \leq|m| \leq l)$, while $a_{l}^{m}$ presents the unknown expansion coefficients. The maximal order of the spherical harmonic expansion is usually defined by the integer parameter called the expansion degree $L$. Consequently, each expansion term $a_{l}^{m}$ and $Y_{l}^{m}$ is characterized by two indices. The subscript $l(l=0,1$, $2, \ldots, L)$ indicates the expansion degree, while the superscript $m(m=-l, \ldots, 0, \ldots ; l)$ indicates the order of spherical harmonics, see Mousa et al. (2008) and Garboczi (2002).

The formal definition of the spherical harmonics is expressed in terms of complex functions $Y_{l}^{m}$, but only real valued functions are used hereafter. They are given by:

$$
Y_{l}^{m}(\theta, \varphi)=\left\{\begin{array}{cl}
\sqrt{2} N_{l}^{m} \cos (m \varphi) P_{l}^{m}(\cos \theta) & \text { if } m>0 \\
N_{l}^{0} P_{l}^{0}(\cos \theta) & \text { if } m=0, \\
\sqrt{2} N_{l}^{|m|} \sin (|m| \varphi) P_{l}^{|m|}(\cos \theta) & \text { if } m<0
\end{array}\right.
$$

where $N_{l}^{|m|}$ is a normalization constant and $P_{l}^{|m|}(\cos \theta)$ presents associated Legendre polynomials.

Development of the spherical harmonics expansion is a computational procedure where the calculation of expansion coefficients $a_{l}^{m}$ is required. The least square method is used for this purpose, where the error $E$ between the actual value $R(\theta, \varphi)$ and the spherical harmonics expansion Eq. (1) is being minimized. Using discrete presentation, the approximation error is estimated by deviations in the set of sampling points $i$ $\left(i=1 \ldots, N_{1} \geq M\right)$. Finally, a minimization problem is expressed as follows: 


$$
E=\min _{a_{l}^{m}}\left(\sum_{i=1}^{N_{1}}\left(R\left(\theta_{i}, \varphi_{i}\right)-\sum_{l=0}^{L} \sum_{m=-l}^{l} a_{l}^{m} Y_{l}^{m}\left(\theta_{i}, \varphi_{i}\right)\right)^{2}\right),
$$

where $Y_{l}^{m}\left(\theta_{i}, \varphi_{i}\right)$ presents the values of harmonic functions in sampling point $i$.

Using matrix notations, the problem in Eq. (3) is transformed to a matrix equation:

$$
[Y][a]=[R],
$$

where $[\boldsymbol{a}] \equiv a_{l}^{m}$ and $[\boldsymbol{R}] \equiv R\left(\theta_{i}, \varphi_{i}\right)$ are column vectors, and rectangular matrix $[\boldsymbol{Y}] \equiv Y_{l}^{m}\left(\theta_{i}, \varphi_{i}\right)$ contains values in sampling points. The solution of this matrix equation is obtained by matrix algebra applying a standard Moor-Penrose pseudo-inversion procedure.

Quality of numerical SH approximation could be also evaluated not only by surface deviations (Eq. (3)) but also by considering integral geometric characteristics of the particle body, including volume and surface. Numerical integration procedure was utilized for calculation purposes. Grid used for calculation of expansion coefficients was explored for these purposes. Four node grid cells are divided into four triangles. As a result, triangle is described by three nodal points 1 , 2 and 3, respectively, and it is defined by three polar radii. Converting between polar and Cartesian coordinates, the polar triangle is transformed to an arbitrary oriented triangle, which geometry is defined by three vectors as follows $\boldsymbol{x}_{1}=\left\{x_{1}, y_{1}, z_{1}\right\}^{\mathrm{T}}, \boldsymbol{x}_{2}=\left\{x_{2}, y_{2}, z_{2}\right\}^{\mathrm{T}}$ and $\boldsymbol{x}_{3}=\left\{x_{3}, y_{3}, z_{3}\right\}^{\mathrm{T}}$. The area of this triangle embedded in the three-dimensional space is expressed as the modulus of the vector product:

$$
S_{t r}=\frac{1}{2}\left|\left(x_{3}-x_{1}\right) \times\left(x_{2}-x_{1}\right)\right| .
$$

Calculation of the volume follows the same path. Each of the surface triangles forms a pyramid where the forth vertex is fixed in the origin of coordinates.
The volume for one pyramid is calculated as:

$$
V_{p i r}=\frac{1}{2} h_{p i r} S_{t r, n} .
$$

Here, $h_{\text {pir }}$ stands for the height of the pyramid, while $S_{t r, n}$ is the normal projection of the area of the surface triangle.

\section{Low-Resolution SH models for Quasi-Quadric particles}

Quasi-quadric particle shape considered in this paper is described by three dimensional parameters $a, b$ and $c$, which are defined as the half of the side lengths of the smallest imaginary box that can contain a particle (Fig. 1(b)). The box for any shape under consideration is centred on the origin of the Cartesian coordinate system and aligned with the directions of their axes. Parameter $c$ means projection of the distance from the origin into direction of axis $\mathrm{Oz}$. Traditionally, $c$ is termed hereafter as length while dimensions in perpendicular directions, $a$ and $b$, are identified to cross-section as breadth and thickness. Taking into account the full three-plane symmetry, one octant of the body limited by positive values of Cartesian coordinate planes $x \geq 0, y \geq 0, z \geq 0$ will be considered numerically. Consequently, modelling domain is mapped into a reduced single angular sub-domain defined by angles $0 \leq \varphi \leq \pi / 2$ and $0 \leq \theta \leq \pi / 2$. The remainder part of the particle surface will be restored using symmetry rules.

The particle shape may be also characterised by elongation, or flattening, through the factor $k_{\text {elon }}=$ $c / a$. The equal sized shape is characterized by elongation ratio $k_{\text {elon }}=1$, i.e. by equal sizes $c=a$. The oblate shape is characterized by the dimensional values $c<$ $a$, i.e. elongation ratio $k_{\text {elon }}<1$. It can be imagined as flattened equal sized shape. The prolate shape characterised with $k_{\text {elon }}>1$ is obtained by extending equal sized shape. a)

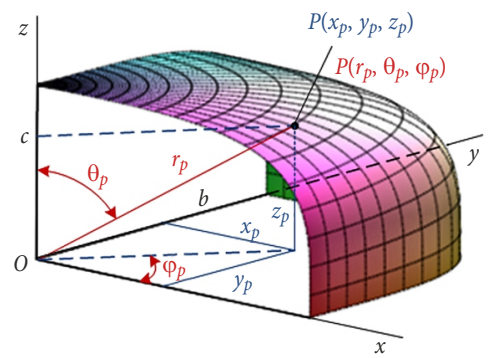

b)

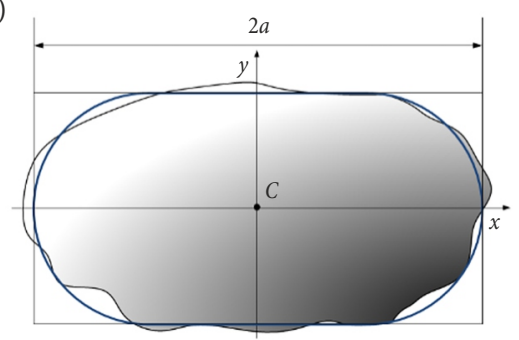

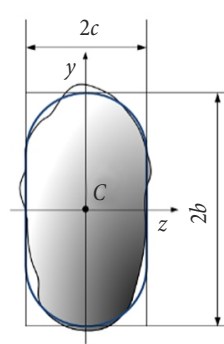

Fig. 1. Illustration of particle geometry: (a) modelling domain on the base of quadric image,

(b) quasi-quadric particle in bounding box 
Two families of quasi-quadric shapes such as quasi-ellipsoids and quasi-prisms, typical in geotechnical engineering, are considered hereafter for illustration purposes. Several reasons could be mentioned to motivate the choice of the above illustrative shapes. Ellipsoid, especially the rotational ellipsoid, is the most simple but the most frequently studied non-spherical smooth analytical shape considering the framework of DEM. It is obvious that the rounded particles of fluvial origin may be classified as quasi-ellipsoids. Industrially treated minerals could be classified as quasi-prisms.

Mathematical relations defined by Eqs (1)(4) present a theoretical framework of SH applicable to the description of particles of arbitrary shape. This LRSH approach involves surface models which may be defined with four complexity levels. The structure of expression Eq. (1) is characterized by expansion degrees $L=0,1,2,3$ containing $M=1,4,9,16$ terms, respectively. The vector of coefficients $\boldsymbol{a}=\left\{a_{l}^{m}\right\}^{T}$ has the following structure $\boldsymbol{a}=\left\{a_{0}^{0}, a_{1}^{-1}, a_{1}^{0}, a_{1}^{1}, a_{2}^{-2}, a_{2}^{-1}, a_{2}^{0}, a_{2}^{2}, a_{3}^{-3}, a_{3}^{-2}, a_{3}^{-1}, a_{3}^{0}, a_{3}^{1}, a_{3}^{2}, a_{3}^{3}\right\}^{T}$, while polynomials $Y_{l}^{m}$ are formed by harmonic functions. Therewith, the general expression of particle surface is simplified by truncated $\mathrm{SH}$ expansion

$$
\begin{aligned}
& F_{L 3}(\theta, \varphi)=a_{0}^{0}\left|Y_{0}^{0}(\theta, \varphi)\right|+\sum_{m=-1}^{1} a_{1}^{m}\left|Y_{1}^{m}(\theta, \varphi)\right|+ \\
& \sum_{m=-2}^{2} a_{2}^{m}\left|Y_{2}^{m}(\theta, \varphi)\right|+\sum_{m=-3}^{3} a_{3}^{m}\left|Y_{3}^{m}(\theta, \varphi)\right|
\end{aligned}
$$

where $F_{L 3}(\theta, \varphi)$ is a resultant function of the lowresolution harmonics model. Explicit expressions of harmonic functions may be found in Garboczi (2002).

Formally, the shape defined by Eq. (7) reflects the initial shape into a unified form. The zero term $(L=0)$ is characterized by a harmonic function $Y_{0}^{0}(\varphi, \theta)=(1 / 2 \sqrt{\pi}) a_{0}^{0}$ describing a sphere. It is easy to persuade, that the coefficient $a_{0}^{0}$ is related to the sphere radius $R$ by the following relationship $a_{0}^{0}=2 \sqrt{\pi R}$. The first order terms $(L=1)$ describes the distortion of the shape in main directions. The geometrical interpretation of the higher order terms $(L=2,3)$ is more complicated.

\section{Numerical illustration of LRSH models}

The mathematical model of LRSH defined by Eq. (7) reflects the mathematical side of the shape descriptors. It is obvious that the variation of 16 undefined coefficients indicates an opportunity to generate a huge number of particular shapes. The suitability of the LRSH model to particular shapes should be proved by conducting numerical experiments.

The performed numerical study involves the generation of quasi-quadric particles by approximation to perfect shapes and evaluation of their properties by applying various indicators. Both of the two shape families, ellipsoids as well as prisms, are represented with discrete number of similar particles characterised by an specific elongation factor $k_{\text {elon }}$, non-dimensional values of which are ranging between 10 and 0.1, i.e. $10 \geq k_{\text {elon }} \geq 0.1$. Each particular particle shape with a fixed value of $k_{\text {elon }}$ is considered as an assembly of "source" particles and set of four "quasi-shaped" particles. The "source" particle presents perfectly shaped particle, while "quasi-shaped particles" by a set of four LRSH models reflecting four expansion degrees $L=0$, $1,2,3$.

The properties of the quasi-shaped particles were examined by comparing the values of selected indicators. Two relative indicators, the relative volume and the relative least square error, were introduced for evaluation purposes. The relative volume $V_{\text {rel, }}\left(k_{\text {elon }}\right)$ is defined as the ratio of the volume of the distorted quasi-shape $V_{\text {quasi, } L}\left(k_{\text {elon }}\right)$ and the volume of "source" particle $V_{\text {source }}\left(k_{\text {elon }}\right)$ :

$$
V_{\text {rel }, L}\left(k_{\text {elon }}\right)=V_{\text {quasi }, L}\left(k_{\text {elon }}\right) / V_{\text {source }}\left(k_{\text {elon }}\right),
$$

where $L$ denotes expansion degree.

The relative error $E_{\text {rel, } L}\left(k_{\text {elon }}\right)$ is defined as least square error density over the surface. It is calculated as the ratio of the total least square error $E_{L}$ defined in Eq. (3) and the surface area of the "source" particle $S_{\text {source }}\left(k_{\text {elon }}\right)$

$$
E_{\text {rel }, L}\left(k_{\text {elong }}\right)=E_{\text {quasi }, L}\left(k_{\text {elong }}\right) / S_{\text {source }}\left(k_{\text {elong }}\right) \text {. }
$$

Suitability of the LRSH models is demonstrated by considering two families of quasi-quadric particles; quasi-ellipsoid and quasi-prisms. Simulations are performed considering an axially symmetric section, where $a=b$. It means that ellipsoids are restricted to rotational, or sphere-sectional, ellipsoids, while prisms are restricted to square-shaped prisms. Simulation results obtained for quasi-ellipsoid are illustrated in Figure 2 while results of quasi-prism are presented in Figure 3. Three separate graphs in each subfigure demonstrate variations of the volume indicator $V_{\text {rel, } L}\left(k_{\text {elon }}\right)$ against elongation factor for specified value of expansion degree $L=1,2,3$. Discrete values of the elonga- 
a)

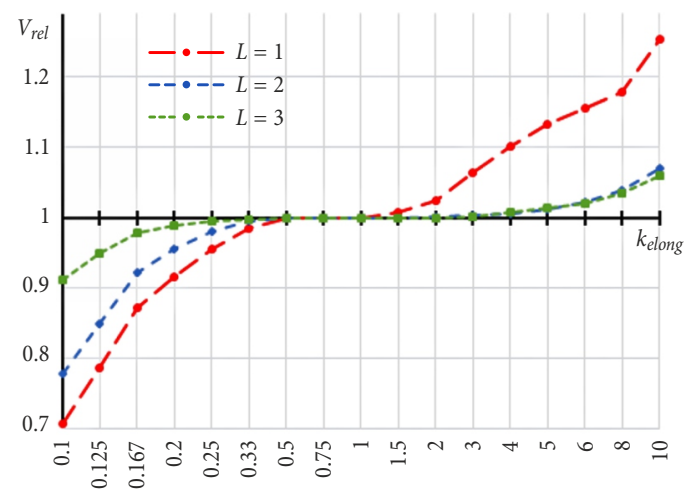

b)

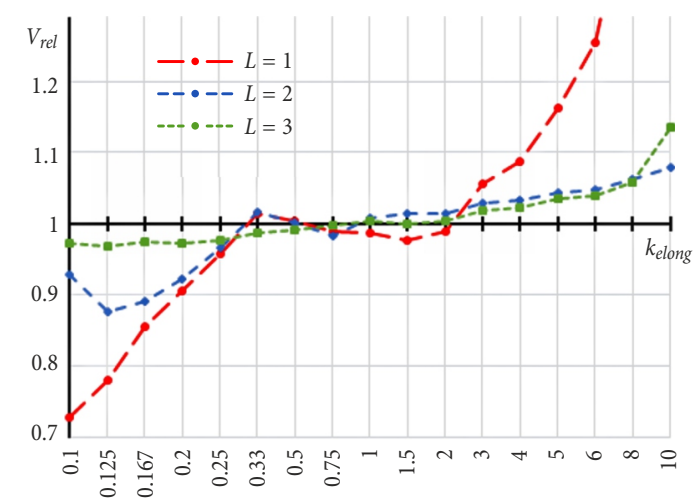

Fig. 2. Variations of relative volumes of elongated (flattened) quasi-quadric particles obtained by LRSH with different values of expansion degree $L:$ a) quasi-ellipsoids; b) of quasi-prisms

a)

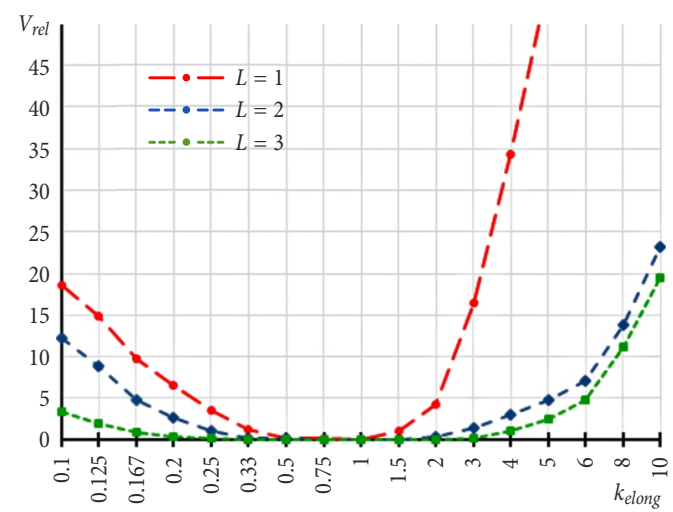

b)

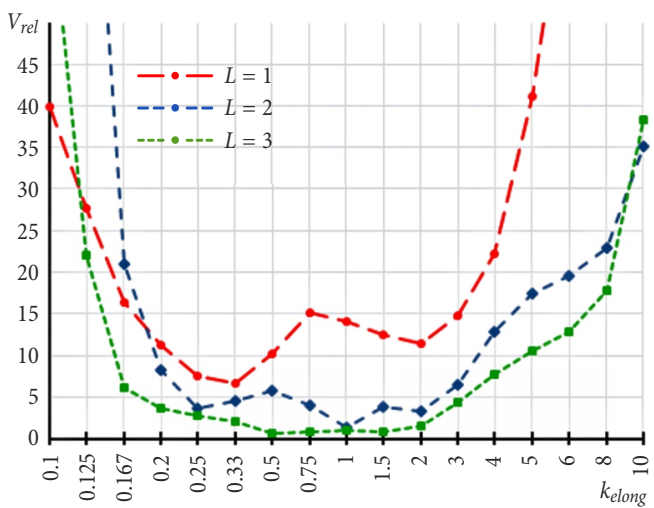

Fig. 3. Variations of square root errors of elongated (flattened) quasi-quadric particles obtained by LRSH with different values of expansion degree $L:$ a) quasi-ellipsoids; b) quasi-prisms

tion factor $k_{\text {elon }}$ marked on the graph are uniformly distributed on horizontal axis, but different scales were used for equidistant location of $k_{\text {elon }}$ values in different segments $k_{\text {elon }}>1$ and $k_{\text {elon }}<1$, respectively. The graph values indirectly reflect the quality of particular LRSH model.

Results show that LRSH approach seems to be able to sufficiently and accurately represent particles. It could be stated that even 10 times elongated or flattened shapes could be described by SH using $L=3$ extension degree quite acceptable (5 percent accuracy). Thereby, different character of approximation trend was observed for elongated and flattened figures as well as for different figures. This could be explained by the fact that different harmonics dominate in different configurations.

Pictorial summary of several selected 3D SH images illustrating essential points is shown in Table 1. Here, three-dimensional views contribute to our understanding. It is easy to detect that the longest particles are characterised by the highest local distortion of particle surface.

For a deeper understanding of character and capabilities of the low-resolution $\mathrm{SH}$, the detailed illustration of the obtained shape profiles is necessary. Consequently, profiles of oblate shaped particles characterised by an elongation factor $k_{\text {elon }}=1 / 4$ will be considered for the sake of illustration. Longitudinal profiles, $y=0$, and cross-sectional profiles, $z=0$, of these particle surfaces are shown in Figure $4 a, c$ and Figure $4 \mathrm{~b}$, d, respectively.

Application of the LRSH to oblate (flattened) shapes discovered some tendencies. Spherical harmonic with the lowest-extension degree $(L=1)$ independently of the initial shape turns into quasi-ellipsoid with a sharp angle in the middle section $z=0$ and wavy profile in the vicinity of the cross-section centre $x=0$, $y=0$. Increase of expansion reduces the above mentioned central almost circularly varying sharpness and improves the global indicators, thereby, approaching to a perfect shape. Approaching tendencies are, however, different. Final errors for the ellipsoid are negligible, and therefore an accurate practically valid shape is obtained. The obtained quasi-prism has remarkable deviations compared to perfect shape. Rounding of sharp edges is a characteristic feature of quasi-prisms. 
Table 1. Pictorial summary of selected shapes

\begin{tabular}{|c|c|c|c|c|}
\hline \multicolumn{3}{|c|}{ Values of expansion degree $L$} \\
\hline$L=1$ & $L=3$ & $L=1$ & $L=3$ \\
\hline \multicolumn{3}{|c|}{ Oblate sphere-sectional quasi-ellipsoid } \\
$k_{\text {elon }}=1 / 8$ \\
\hline
\end{tabular}

\section{Local effects and contact behaviour}

Characterisation of surfaces of non-spherical particles using of continuous representation functions by Eq. (1) or evaluation of integral parameters such as surface or volume (Eqs (5) and (6)) reflects only a part of the entire problem. In certain situations, local variations of the surface may have significant contribution to particle properties, especially in describing the contact behaviour. The need to evaluate local effects in LRSH models is also inspirited by the earlier results presented in Table 1 and Figures 2, 3 and 4. Therefore, a mathematically consistent description of the surface comprising principal curvatures, their principal directions and other parameters containing surface derivatives is essential.

For numerical illustration of the contact behaviour, the shape sample consisting on a rotational oblate ellipsoid defined by size parameters $a=b=2$ and $c=1$ will be investigated. Curvatures and calculation of the normal contact force are evaluated. For this, analytical instead of numerical treatment of the contact will be applied while numerical FEM analysis will be also used for verification purposes. Regarding axial symmetry geometry of the particle is defined by the surface profile in longitudinal section. The perfect analytically described ellipsoid and quasi-ellipsoids obtained by low-resolution SH approximation $L=1$ is considered for representation of this shape. Various profiles of particle surface plotted in the Cartesian $O x z$ plain are shown in Figure 5a.

In order to restore full picture of particle properties, these profiles $r(\theta)$ are also alternatively considered in spherical coordinates at fixed polar angle $\varphi=0$ and depicted in Figure 5b. Profile of quasi-ellipsoid is originally calculated by LRSH approximation using Eq. (7). Here, perfect ellipsoid is illustrated by thin solid line while SH model by dashed lines.

Comparing character of perfect and $\mathrm{SH}$ profiles, three characteristic regions could be detected. Here, the conditional boundaries $\theta_{1}=0.143 \pi$ and $\theta_{2}=0.052 \pi$ are denoted in Figure 5 by dots. The middle region between $\theta_{1}$ and $\theta_{2}$ illustrates that approximated profile quite closely mimics analytical one. The first region $(0$ $\leq \theta \leq \theta_{1}$ ) restricted by angle $\theta_{1}$ indicates occurrence of wavy profile in vicinity of the cross-section centre $x=0, y=0$. The third region $\left(\theta_{2} \leq \theta \leq \pi / 2\right)$ restricted by angle $\theta_{2}$ indicates that $\mathrm{SH}$ produces asperity with sharp angle in the central section $z=0$. 
a)

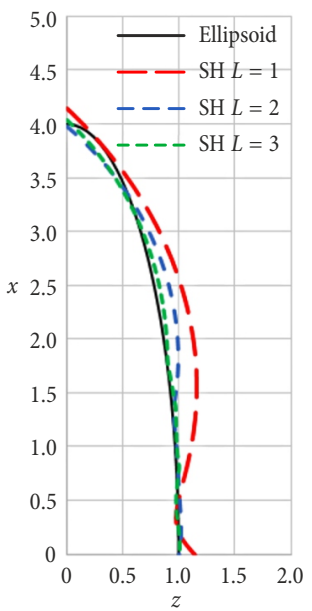

b)

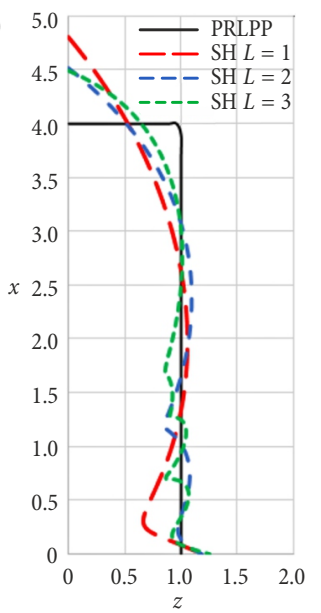

c)

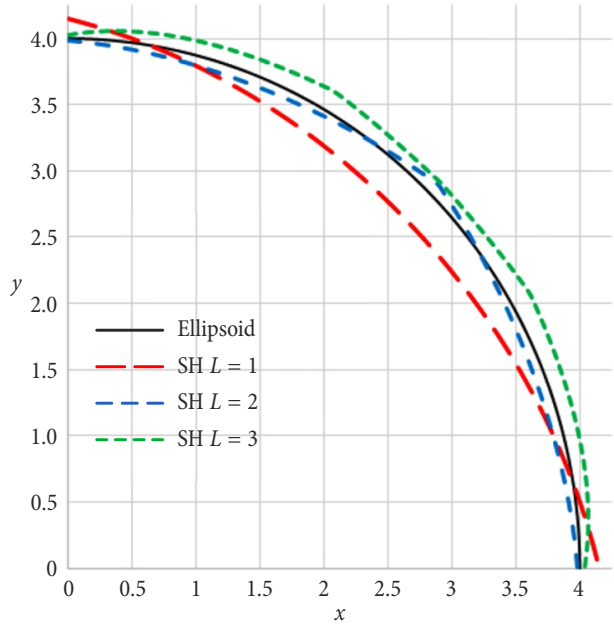

d)

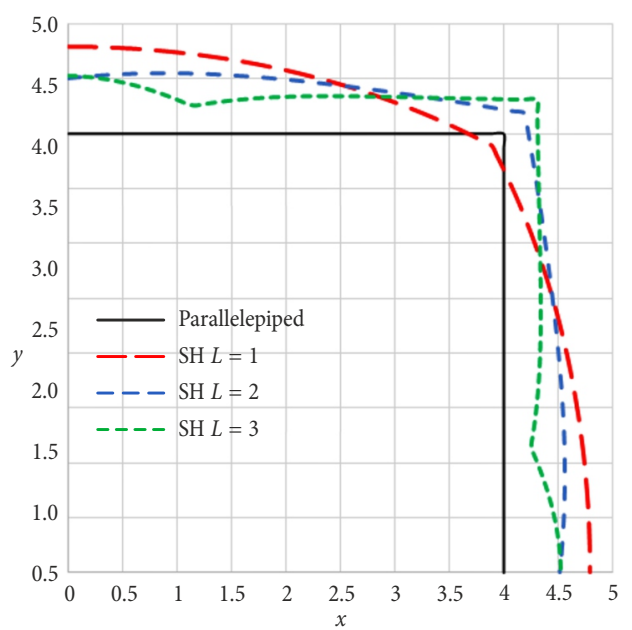

Fig. 4. Cartesian profiles of flattened $\left(k_{\text {elon }}=1 / 4\right)$ particles obtained by spherical harmonics with different $(L=1, L=2$, $L=3$ ) expansion degrees: longitudinal profiles (a) and crosssectional profiles (c) of quasi-ellipsoid; longitudinal profiles

(b) and cross-sectional profiles (d) of quasi-prism

If consideration of the non-smooth and concave surface is not satisfactory, the continuous not necessary smooth quasi-quadric surface could be constructed. In this case, both the first and the third regions are a)

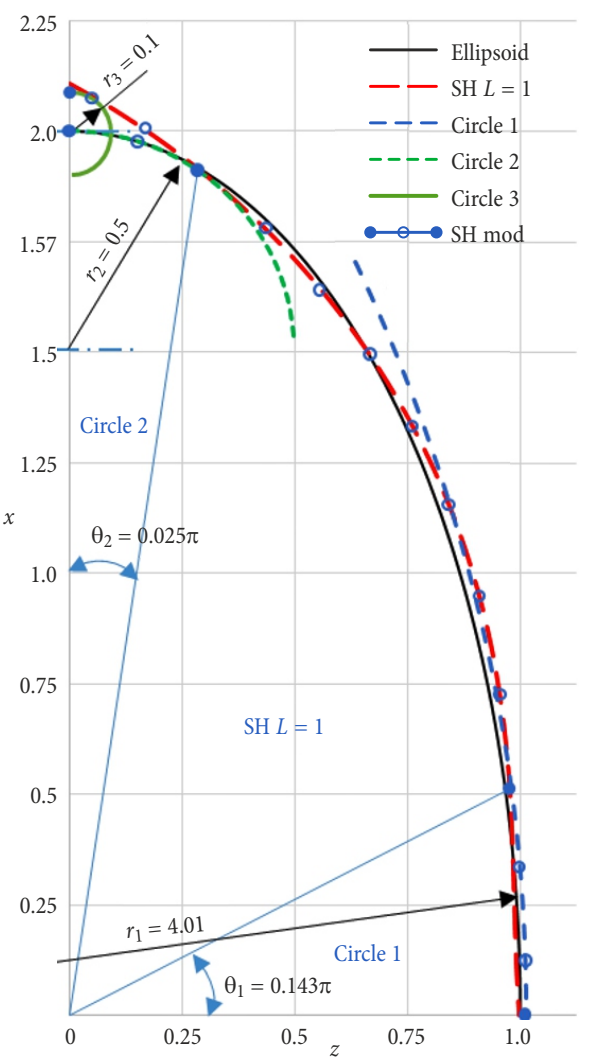

b)

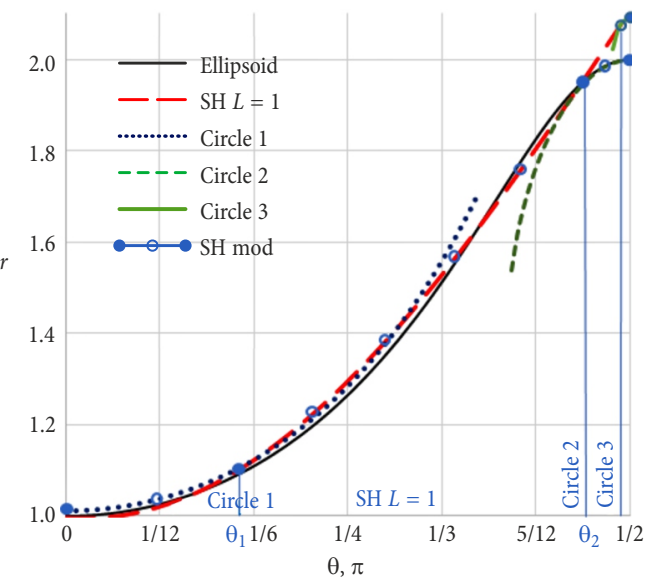

Fig. 5. Profiles and curvatures of quasi-quadric surfaces (quasi-ellipsoids): a) in Cartesian coordinates, b) in spherical coordinates

replaced by circles having common tangents, or common external normals, in intersection point's $\theta_{1}$ and $\theta_{2}$. In the first region, the centre of the approximating circle 1 is located in the central axis $O z$, while its radius $r_{1}$ is larger than semi-axis $c$, i.e. $r_{1}>c$. Increasing of approximation order reduces the boundary angle $\theta_{1}$, and, consequently, the size of region. In three dimensions, this region presents spherical cup with radius $r_{1}$.

In the third region, the approximating circle 2 is an inscribed circle which centre is located in the central plane Oxy (initial radius $r_{2}=0.5$ ). Reducing this 
radius $r_{2}$ and approaching it to the almost zero, a set of surfaces with the new radii $r_{3} \rightarrow$ min, approximating quasi-quadric shape with different degree of accuracy may be generated. Increase of expansion reduces the above-mentioned central almost circularly varying sharpness and improves the global indicators, thereby, approaching to perfect shape.

In three dimensions, this region corresponds to a toroid, or spherical ring, with cross-sectional radius $r_{2}$ and external radius $\approx a$. In summary composite enveloping $\mathrm{SH}$ surface is generated. Three-dimensional views of particles are illustrated in Figure 6, where quasi-ellipsoid with sharp edge and resultant quasiquadric composite bodies are shown in Figure 6(a) and $6(\mathrm{~b})$, respectively.

It could be noted, that the approximation of complex curves by circular arches is a common practice used in DEM. The equivalent contact sphere method for approximation of ellipsoids by four arches is elaborated in Johnson et al. (2004). Approximation of rotational prolate ellipsoid by the spherical end cup combined with the multi-sphere technique in the reminder body is presented in Markauskas et al. (2010).

An important characteristic of particle geometries that contributes to particle contacts are particle curvatures. A general method for obtaining expressions for curvatures in terms of surface derivatives is provided in differential geometry while detailed description of curvature expressions for ellipsoid are given by Harris (2006) and Poelaert et al. (2011) will be explored for further analysis of contact of quasi-ellipsoids. Differentiation of $\mathrm{SH}$ and analysis of ellipsoid's curvatures were considered by Garboczi (2002), while a more recently applied methodology is given in Radvilaite et al. (2017).

a)

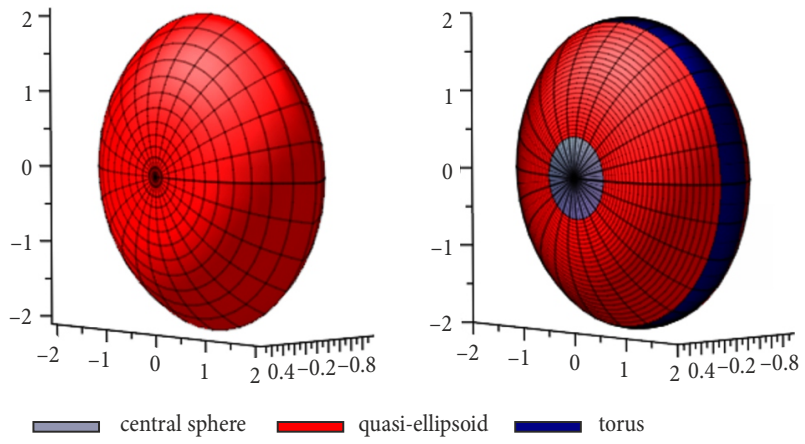

Fig. 6. Three-dimensional views of particles: a) quasi-ellipsoid with sharp edge, b) resultant quasi-quadric composed various bodies
Variations of curvature radii are shown in Figure 7 . The obtained radii or curvatures are used further for calculation of normal contact force. Potentially, analytical instead of numerical approach to calculation of the contact would be preferable.

It is useful to remind that contacts evaluation is the mostly time consuming algorithmic step when performing DEM simulations. Omitting conceptual discussion on detection of contact point for non-spherical particles, which was comprehensively described in the review papers of Lu et al. (2015) and Zhong et al. (2016), the recent paper addresses the calculation of the contact forces.

Analytical treatment of normal contacts for continuous solid contact partners $i$ and $j$ is based on the elastic contact theory of Hertz (Johnson 1987). The state of the contact behaviour between ellipsoidal particles is reviewed and discussed by Zheng et al. (2013). It was stated that regardless of the specific particle shape, two particles loaded in the normal direction touch and deform each other over an elliptical area. Consequently, considering of contacts between nonspherical particles, the normal contact force can be expressed by modifying the conventional Hertz force $F_{i j H n}=F_{H n}$ which is related to the inter-particle overlap (displacement) $u$ as follows:

$$
F_{H n}=\frac{4}{3} c_{c o r}\left(\rho_{i 1}, \rho_{i 2}, \rho_{j 1}, \rho_{j 2}\right) E_{e f f} \sqrt{\rho_{e f f} u^{3 / 2}},
$$

where, the effective elastic modulus is expressed in terms of Poisson's ratios $v_{i}$ and $v_{j}$ and elasticity moduli $E_{i}$ and $E_{j}$ of contacting partners by the relation $1 / E_{e f f}=\left(1-v_{i}^{2}\right) / E_{i}+\left(1-v_{j}^{2}\right) / E_{j}$.

Both of two other parameters - the effective radius of curvature:

$\rho_{\text {eff }}\left(\rho_{i 1}, \rho_{i 2}, \rho_{j 1}, \rho_{j 2}\right)=$

$1 /\left(2 \sqrt{A\left(\rho_{i 1}, \rho_{i 2}, \rho_{j 1}, \rho_{j 2}\right) B\left(\rho_{i 1}, \rho_{i 2}, \rho_{j 1}, \rho_{j 2}\right)}\right)$,

and non-dimensional correction factor:

$$
\begin{aligned}
& c_{c o r}\left(\rho_{i 1}, \rho_{i 2}, \rho_{j 1}, \rho_{j 2}\right)= \\
& \left.1-\left[\left(\frac{B\left(\rho_{i 1}, \rho_{i 2}, \rho_{j 1}, \rho_{j 2}\right)}{A\left(\rho_{i 1}, \rho_{i 2}, \rho_{j 1}, \rho_{j 2}\right)}\right)^{0,0684}-1\right]^{1,531}\right]^{-\frac{3}{2}} .
\end{aligned}
$$

Eq. (12) is a simplification and approximation of that proposed by Hale, see Zheng et al. (2013), where $A$ and $B$ are new variables determined from the local geometry (Johnson 1987). 
a)

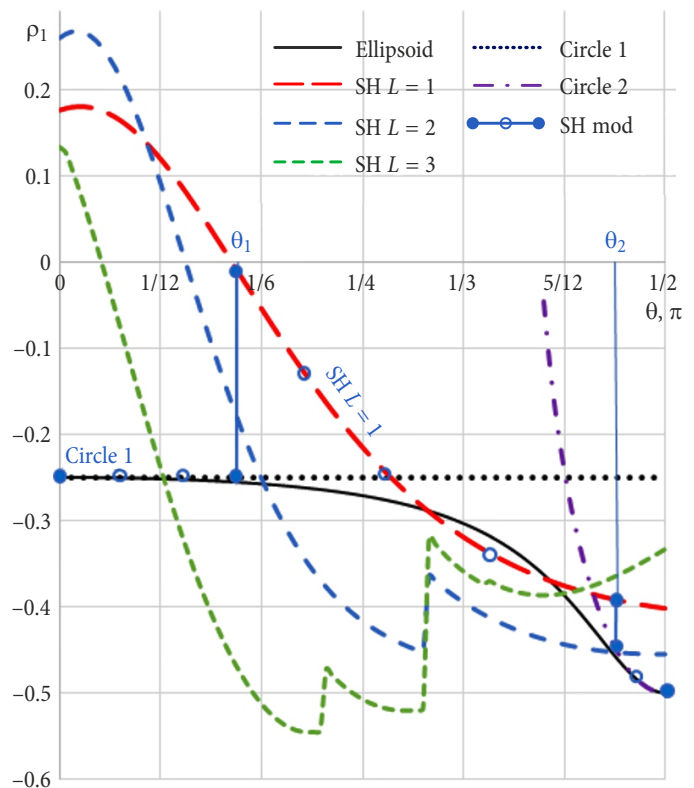

b)

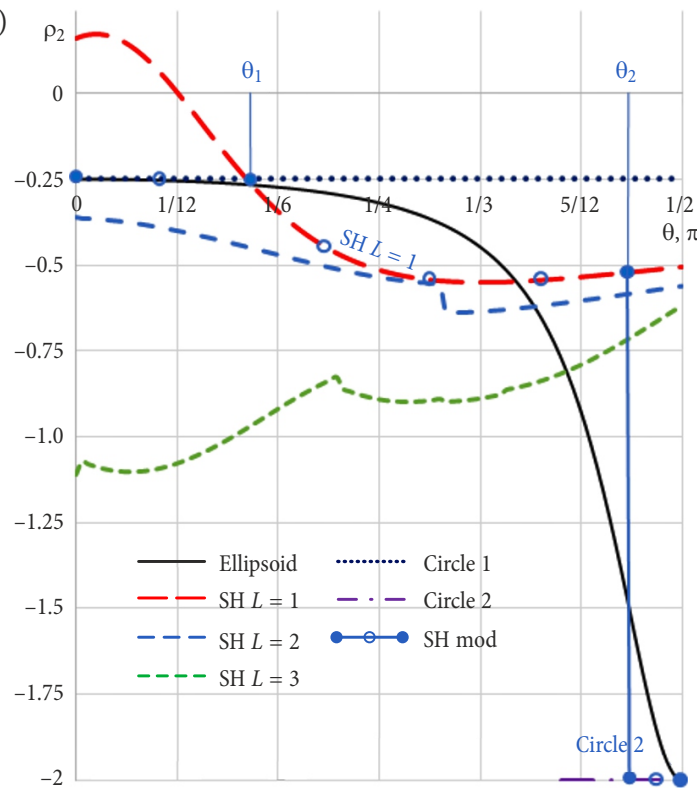

Fig. 7. Variation of curvature radii in longitudinal section presented in spherical coordinates a) radius $\rho_{1}$ normal to section profile, b) radius $\rho_{2}$ perpendicular to cross-section profile

Contribution of the local particle shape occurred due to LRSH description is demonstrated by solving the normal contact of the above oblate ellipsoid with a plane plate. The case of contact of a perfectly elastic ellipsoid onto a semi-infinite plane surface was also considered in Wynn (2009).

Computational set up of the normal particleplane contact is illustrated in Figure 8.

Here, normal orientation of the particle with a plane perpendicular to the central axis $O z$ is shown. The particle centre was subjected by a normal displacement $u$, while contact force $F_{n}$ is the resultant reaction.

Analytical treatment of the above contact is performed by applying Eqs (10)-(14). Parameters of the

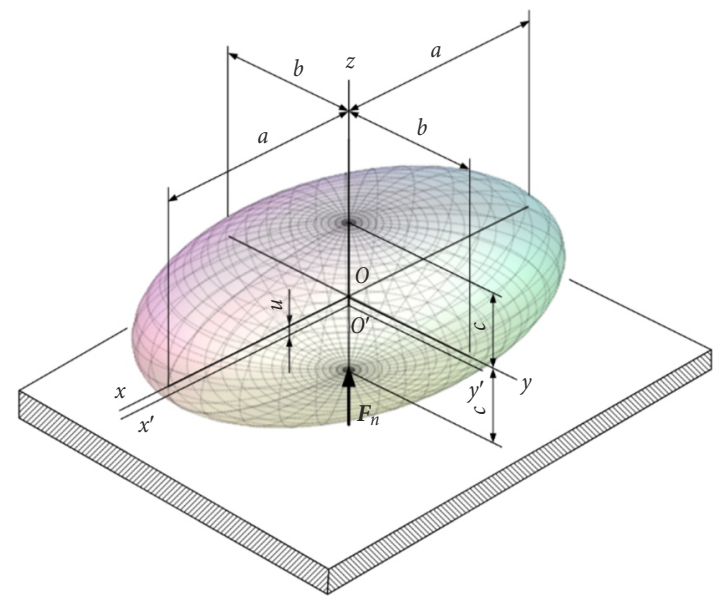

Fig. 8. Computational set up of the normal particle-plane contact body $i$ are identified to ellipsoid denoted as ell, while second body $j$ is identified as plane and denoted with the subscript $p l$.

In this problem, for plane curvature radii $\rho_{p l 1}=$ $\rho_{p l 2}=\infty$, consequently, parameters $A \equiv A\left(\rho_{\text {ell } 1}\right)$ and $B \equiv B\left(\rho_{\text {ell2 }}\right)$ take a form:

$$
A\left(\rho_{\text {ell } 1}\right)=\frac{1}{2 \rho_{\text {ell } 1}} \text { and } B\left(\rho_{\text {ell } 2}\right)=\frac{1}{2 \rho_{\text {ell } 2}} \text {. }
$$

It is easy to detect that these parameters are curvature parameters $A=\kappa_{\text {ell } 1} / 2$ and $B=\kappa_{\text {ell } 2} / 2$.

Variations of correction factor in longitudinal profile against azimuthal angle $\theta$ are given Figure 9, where about $6 \%$ increase of the load is observed with increased curvatures.

Comparison of various approaches is carried out in non-dimensional form, and computational experiment is organised in the following manner. All length variables are scaled to parameter $R$ and defined by a set of non-dimensional parameters. Therewith, defining indentation displacement by $u_{r e l}=u / R$ and effective radius by $\rho_{\text {eff rel }}=\rho_{\text {eff }} / R$, the normal force in Eq. (10) may be also expressed in terms of non-dimensional and dimensional parameters as

$$
F_{H n}=F_{H n \text { rel }} E_{\text {eff }} R^{2},
$$

where contact force is defined by a non-dimensional factor:

$$
F_{\text {Hn rel }}=\frac{4}{3} c_{\text {cor }} \sqrt{\rho_{\text {eff rel }}}\left(u_{\text {rel }}\right)^{3 / 2} \text {. }
$$




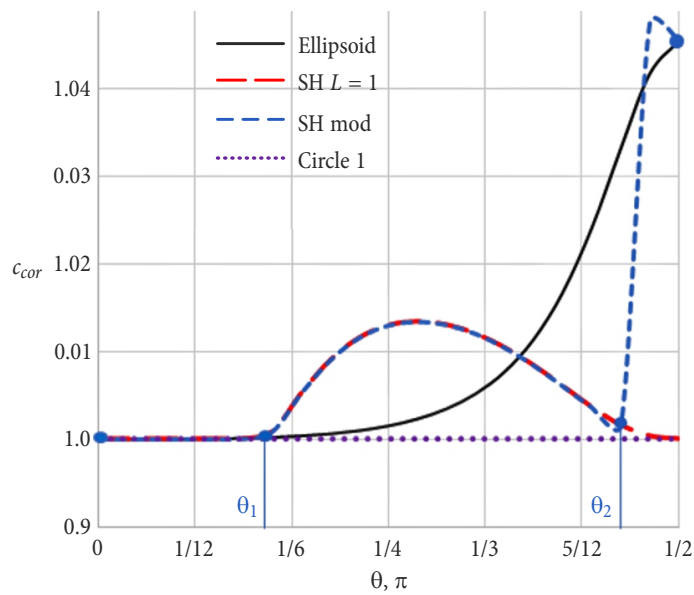

Fig. 9. Variations of correction factor $c_{c o r}$ for particle-plane contact in longitudinal section

Contact analysis was conducted by relating the dimensional length parameter $R$ with the size of ellipsoid, $R=b$, and assuming the value of the relative indentation $u_{\text {rel }}=0.01$. Four characteristic samples were solved analytically, while sample data and solution results are given in Table 2. Each of the samples are denoted by the specified identifier, name of which containing orientation of the contacting particle, contact type and solution method. The first two samples denoted as RAD_ELL_ANL and RAD_SH_ANL illustrate results of radially oriented perfect ellipsoid and SH model with circular round off of artificial asperity, respectively. The next two samples denoted as NORM_ ELL_ANL and NORM_SH_ANL illustrate results of normally oriented perfect ellipsoid and SH model with spherical cup, respectively. In order to verify applicability of analytical models, the problem of normal elastic particle-plane contact was solved numerically, using the finite element method (FEM). The non-adhesive perfectly stick contact is assumed, therefore, tangential sliding is not allowed. The surfaces of contacting bodies in contact zone are assumed to be smooth. The problem is three-dimensional, and computational domain comprises a quarter of the bodies because of its plane symmetry. Contacting bodies are discretised by the volume tetrahedral-type elements defined by 8 nodes. The multipurpose commercial available FEM package ANSYS (ANSYS 2016) was used for these purpose.

Three characteristic samples were solved numerically. Two samples of radially oriented ellipsoid denoted as RAD_ELL_FEM and RAD_SH_FEM involves computations of radially oriented perfect ellipsoid and SH model with sharp edge, respectively. The third sample NORM_ELL_FEM involves normally oriented ellipsoid.

Illustration of computational results is presented in Figure 10. Here, numerically obtained distributions of the von Mises stresses are shown.

The computed values of the normal contact force correspond to expected results. The three samples of normal contact present actually spherical contacts, the difference between analytical Hertz and the FEM results is characterised by $0.3 \%$ error. Generally, results illustrated and confirmed that concave surface could be modified by spherical cup.

For evidence of models contribution, the values of the normalised force factor $F_{\text {rel }} / F_{\text {relmax }}$ is introduced and presented in the last column in Table 2. The most interesting results are obtained by considering radial contact where dramatically increasing capacity of penetration with sharpening of end cup is demonstrated. Summarising contact experiment it could be stated that LRSH could be used for large variety of quasiquadric shapes.

Table 2. Summary of contact analysis results

\begin{tabular}{|c|c|c|c|c|c|c|c|c|}
\hline \multirow{2}{*}{$\begin{array}{l}\text { Model } \\
\text { No }\end{array}$} & \multirow{2}{*}{$\begin{array}{c}\text { Model } \\
\text { identification }\end{array}$} & \multirow{2}{*}{$\begin{array}{l}\text { Orientantion } \\
\text { angle } \Theta\end{array}$} & \multicolumn{2}{|c|}{ Curvature radii } & \multicolumn{2}{|c|}{ Non-dimentional factors } & \multicolumn{2}{|c|}{ Relative forces } \\
\hline & & & $\bar{\rho}_{n}=\rho_{n} / \rho$ & $\bar{\rho}_{t}=\rho_{t} / \rho$ & $c_{c o r}$ & $\rho_{\text {eff rel }}$ & $F_{r e l}$ & $F_{\text {rel }} / F_{\text {rel max }}$ \\
\hline \multicolumn{9}{|c|}{ Analytical solutions } \\
\hline 1 & RAD_ELL_ANL & $\pi / 2$ & 0.25 & 1.0 & 1.0455 & 0.5 & $0.986 \cdot 10^{-3}$ & 0.522 \\
\hline 2 & RAD_SH_ANL & $\pi / 2$ & 0.05 & 1.0 & 1.1782 & 0.2236 & $0.743 \cdot 10^{-3}$ & 0.3935 \\
\hline 3 & NORM_ELL_ANL & 0 & 2.0 & 2.0 & 1 & 2.0 & $1.885 \cdot 10^{-3}$ & 0.998 \\
\hline 4 & NORM_SH_ANL & 0 & 2.005 & 2.005 & 1 & 2.005 & $1.888 \cdot 10^{-3}$ & 1.0 \\
\hline \multicolumn{9}{|c|}{ Numerical (FEM) solutions } \\
\hline 5 & RAD_ELL_FEM & $\pi / 2$ & 0.25 & 1.0 & - & - & $0.707 \cdot 10^{-3}$ & 0.3745 \\
\hline 6 & RAD_SH_FEM & $\pi / 2$ & - & 1.0 & - & - & $1.936 \cdot 10^{-4}$ & 0.1025 \\
\hline 7 & NORM_ELL_FEM & 0 & 2.0 & 2.0 & - & - & $1.879 \cdot 10^{-3}$ & 0.995 \\
\hline
\end{tabular}


a)

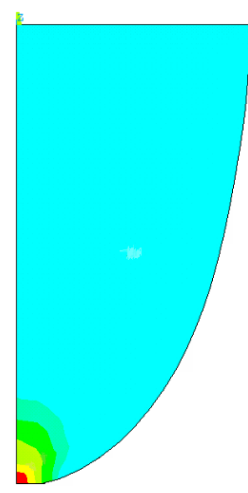

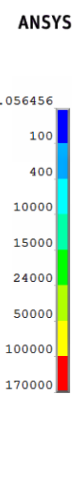

b)

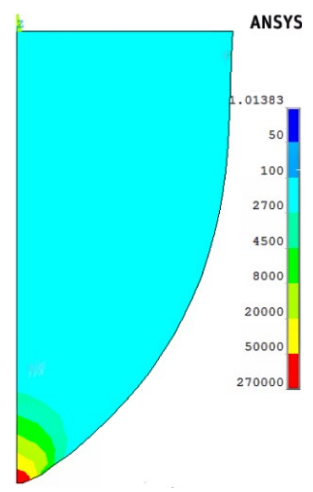

c)

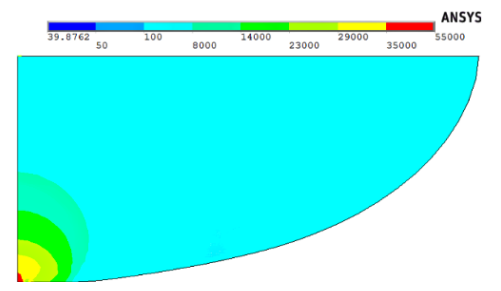

Fig. 10. Variation of von Mises stresses in contact zone of particle at relative indentation $u_{\text {rel }}=0.01$ a) radially oriented perfect ellipsoid (RAD_ELL_FEM); b) radially oriented SH L = 1 model with sharp edge (RAD_SH_FEM); c) normally oriented perfect ellipsoid (NORM_ELL_FEM)

\section{Conclusions}

A semi-analytical approach based on the low-resolution spherical harmonics (LRSH) technique limited with the expansion degree $L \leq 3$ was elaborated. Then the suitability of this technique for the description of non-spherical quasi-quadric shapes, i.e. slightly distorted monomeric shapes of soil particles was investigated. The quality of the results was numerically proved by comparing the least square approximation errors, considering the values of volumes and particle surfaces. It was shown, that the even the simplest case of the suggested low-resolution harmonics technique with $L=1$ showed a good performance. The highest accuracy was achieved for equal-sized shapes but decreased with flattening or elongation of the shapes. Therefore, the suitability of this method to particular shapes should be rechecked. It was also found that the standard differentiation and calculation of curvatures using LRSH may yield to local distortions and angularities. Threfore, enhanced analysis of the local surface gradients and curvatures need to be developed.

\section{References}

ANSYS 2016. ANSYS Version 14.5. [online], [cited 19 July 2016]. Available from Internet: www.ansys.com

Blott, S. J.; Pye, K. 2008. Particle shape: a review and new methods of characterization and classification, Sedimentology 55: 31-63.

Bullard, J. W.; Garboczi, E. J. 2013. Defining shape measures for 3D star-shaped particles: sphericity, roundness, and dimensions, Powder Technology 249: 241-252.

https://doi.org/10.1016/j.powtec.2013.08.015

Cleary, P. W. 2008. The effect of particle shape on simple shear flows, Powder Technology 179(3): 144-163.

https://doi.org/10.1016/j.powtec.2007.06.018
Feinauer, J.; Spettl, A.; Manke, I.; Strege, S.; Kwade, A.; Pott, A.; Schmidt, V. 2015. Structural characterization of particle systems using spherical harmonics, Materials Characterization 106: 123-133. https://doi.org/10.1016/j.matchar.2015.05.023

Garboczi, E. J. 2011. Three dimensional shape analysis of JSC-1A simulated lunar regolith particles, Powder Technology 207(13): 96-103. https://doi.org/10.1016/j.powtec.2010.10.014

Garboczi, E. J. 2002. Three-dimensional mathematical analysis of particle shape using X-ray tomography and spherical harmonics: application to aggregates used in concrete, Cement and Concrete Research 32: 1621-1638. https://doi.org/10.1016/S0008-8846(02)00836-0

Harris, W. F. 2006. Curvature of ellipsoids and other surfaces, Ophthalmic and Physiological Optics 26(5): 497-501. https://doi.org/10.1111/j.1475-1313.2006.00382.x

Johnson, K. L. 1987. Contact mechanics. 1st ed. Cambridge University Press.

Johnson, S.; Williams, J. R.; Cook, B. 2004. Contact resolution algorithm for an ellipsoid approximation for discrete element modeling, Engineering Computations 21(2/3/4): 215-234.

Johnson, S. M.; Williams, J. R. 2009. Sub-discretized surface model with application to contact mechanics in multi-body simulation, Powder Technology 193(3): 319-331. https://doi.org/10.1016/j.powtec.2009.03.006

Kock, I.; Huhn, K. 2007. Influence of particle shape on the frictional strength of sediments - a numerical case study, Sedimentary Geology 196(1-4): 217-233. https://doi.org/10.1016/j.sedgeo.2006.07.011

Lu, G.; Third, J. R.; Müller, C. R. 2015. Discrete element models for non-spherical particle systems: from theoretical developments to applications, Chemical Engineering Science 127: 425-465. https://doi.org/10.1016/j.ces.2014.11.050

Markauskas, D.; Kačianauskas, R.; Džiugys, A.; Navakas, R. 2010. Investigation of adequacy of multi-sphere approximation of elliptical particles for DEM simulations, Granular Matter 12(1): 107-123. https://doi.org/10.1007/s10035-009-0158-y

Masad, E.; Saadeh, S.; Al-Rousan, T.; Garboczi, E.; Little, D. 2005. Computations of particle surface characteristics using optical and X-ray CT images, Computational Materials Science 34: 406-424. https://doi.org/10.1016/j.commatsci.2005.01.010 
Mousa, M.-H.; Chaine, R.; Akkouche, S ; Galin, E. 2008. Toward an efficient triangle-based spherical harmonics representation of 3D objects, Computer Aided Geometric Design 25(8): 561-575. https://doi.org/10.1016/j.cagd.2008.06.004

Nouguier-Lehon, C.; Cambou, B.; Vincens, E. 2003. Influence of particle shape and angularity on the behaviour of granular materials: a numerical analysis, International Journal for Numerical and Analytical Methods in Geomechanics 27(14): 1207-1226. https://doi.org/10.1002/nag.314

Parafiniuk, P.; Molenda, M.; Horabik, J. 2014. Influence of particle shape and sample width on uniaxial compression of assembly of prolate spheroids examined by discrete element method, Physica A: Statistical Mechanics and its Applications 416: 279-289. https://doi.org/10.1016/j.physa.2014.08.063

Poelaert, D.; Schniewind, J.; Janssens, F. 2011. Surface Area and Curvature of the general Ellipsoid, arXiv:1104.5145 [math. CA], (1): 12 .

Radvilaite, U.; Kačianauskas, R.; Rusakevičius, D. 2017. Investigation of symmetric non-spherical particle shapes by applying low-resolution spherical harmonics, Nonlinear Analysis: Modelling and Control 22: 115-133. https://doi.org/10.15388/NA.2017.1.8

Radvilaitè, U.; Ramírez-Gómez, Á.; Kačianauskas, R. 2016. De- termining the shape of agricultural materials using spherical harmonics, Computers and Electronics in Agriculture 128: 160-171. https://doi.org/10.1016/j.compag.2016.09.003

Santamarina, J.; Cho, G. 2004. Soil behaviour: the role of particle shape, in R. J. Jardine, D. M. Potts, K. G. Higgins (Eds.). Advances in Geotechnical Engineering. Proceedings of the Skempton Conference. Thomas Telford, 1-14.

Wynn, E. J. W. 2009. Simulations of rebound of an elastic ellipsoid colliding with a plane, Powder Technology 196(1): 62-73. https://doi.org/10.1016/j.powtec.2009.07.004

Zhong, W.; Yu, A.; Liu, X.; Tong, Z.; Zhang, H. 2016. DEM/ CFD-DEM Modelling of non-spherical particulate systems: theoretical developments and applications, Powder Technology 302: 108-152.

https://doi.org/10.1016/j.powtec.2016.07.010

Zhou, B.; Wang, J.; Zhao, B. 2015. Micromorphology characterization and reconstruction of sand particles using micro $\mathrm{X}$-ray tomography and spherical harmonics, Engineering Geology 184: 126-137. https://doi.org/10.1016/j.enggeo.2014.11.009

Zheng, Q.; Zhou, Z.; Yu, A. 2013. Contact forces between viscoelastic ellipsoidal particles, Powder Technology 248: 25-33. https://doi.org/10.1016/j.powtec.2013.03.020

Urtė RADVILAITE். PhD student at the Department of Strength of Materials and Engineering Mechanics, Vilnius Gediminas Technical University, Lithuania. Research interests: spherical harmonics, granular materials, mathematical modelling.

Álvaro RAMíREZ-GÓMEZ. Assoc. Prof. Dr Department of Mechanical, Chemical and Industrial Design Engineering, Technical University of Madrid, Spain. Research interests: Discrete element method, particle shape models, particle flow experiments, modelling of silo.

Arūnas JARAS. Assoc. Prof. Dr Department of Strength of Materials and Engineering Mechanics, Vilnius Gediminas Technical University, Lithuania. Research interests: Finite element analysis, investigation of buildings of cultural heritage.

Rimantas KAČIANAUSKAS. Prof. Dr Habil. Institute of Mechanics, Vilnius Gediminas Technical University, Lithuania. Research interests: Computational mechanics, modelling of structures and materials, fracture mechanics, coupled problems, finite element method, discrete element method.

Dainius RUSAKEVIČIUS. Assoc. Prof. Dr Department of Strength of Materials and Engineering Mechanics, Vilnius Gediminas Technical University, Lithuania. Research interests: Computational mechanics, finite element method, optimization methods. 